\title{
Musée et troubles du spectre autistique
}

Museums and autistic spectrum disorder

\section{Cyrielle Leriche}

\section{(2) OpenEdition \\ Journals}

Édition électronique

URL : http://journals.openedition.org/cel/4788

DOI : $10.4000 /$ cel.4788

ISSN : 2262-208X

\section{Éditeur}

École du Louvre

\section{Référence électronique}

Cyrielle Leriche, "Musée et troubles du spectre autistique », Les Cahiers de l'École du Louvre [En ligne], 14 | 2019, mis en ligne le 02 décembre 2019, consulté le 10 décembre 2020. URL : http:// journals.openedition.org/cel/4788; DOI : https://doi.org/10.4000/cel.4788

Ce document a été généré automatiquement le 10 décembre 2020.

\section{(c) $($ ) (3) $९$}

Les Cahiers de l'École du Louvre sont mis à disposition selon les termes de la licence Creative Commons Attribution - Pas d'Utilisation Commerciale - Pas de Modification 4.0 International. 


\title{
Musée et troubles du spectre autistique
}

\author{
Museums and autistic spectrum disorder
}

Cyrielle Leriche

1 Depuis les années 1980-1990¹, les musées n'ont cessé d'améliorer le confort de visite et de considérer le visiteur comme une priorité. De nombreux efforts d'accessibilité ont été réalisés pour faciliter la mobilité des personnes non ou malvoyantes ou bien souffrant d'un handicap moteur. Dans le cas de personnes atteintes de déficiences psycho-cognitives, le défi s'avère d'une autre nature. On note, en effet, de multiples pathologies qui peuvent entraver la venue au musée des personnes concernées (personnes ayant des TOC ou encore un syndrome de Gilles de la Tourette etc.). Ces pathologies peuvent induire des conséquences comportementales qui empêchent ces visiteurs de profiter pleinement du musée, tant vis-à-vis d'eux-mêmes que de la gêne occasionnée pour les autres visiteurs : le musée étant avant tout un lieu de sociabilité publique avec un comportement normé attendu. Cela pose en particulier un problème d'adaptation pour les personnes (enfants et adultes) ayant ce que les spécialistes appellent aujourd'hui, des troubles du spectre autistique. En effet, les notions d'autisme et de musée semblent, au premier abord, parfaitement antithétiques. La première se définissant par un repli sur soi, des intérêts intellectuels restreints, voire obsessionnels et la seconde, par l'ouverture sur le monde et sa richesse culturelle.

2 Pourtant ces deux mondes qui semblent vraiment différents, sont-ils si inconciliables? Aux États-Unis et au Canada ${ }^{2}$, notamment, certains musées ont tenté différentes approches afin de montrer que l'intégration de l'autisme au monde muséal n'était pas impossible et surtout que ces approches pouvaient contribuer à une meilleure intégration des personnes dites autistes dans le tissu social. En France, ce type d'expérience se heurte à certaines résistances et à des réticences qui émanent le plus souvent d'une méconnaissance profonde de ce qu'est l'autisme. En effet, l'intégration des personnes atteintes d'autisme apparaît comme extrêmement complexe et beaucoup de questions se posent d'une part sur l'intérêt réel pour ces visiteurs «atypiques » 
d'aller au musée, et d'autre part, sur la complexité des moyens à mettre en œuvre pour favoriser de telles visites.

3 C'est pourquoi il est indispensable dans un premier temps de comprendre ce que ce trouble représente afin de déterminer les principaux facteurs qui empêchent l'accès au musée. Dans un second temps, il sera nécessaire de faire état des diverses expériences menées à travers le monde qui tentent une approche muséale vis-à-vis des autistes et leurs entourages proches (parents et encadrants). Déjà, les résultats de ce premier référencement laissent penser que d'autres projets peuvent être mis en place. C'est ainsi que des visites organisées au Musée du Louvre en 2018 autour de l'environnement sensoriel, ont permis d'identifier un certain nombre de difficultés éprouvées par des personnes autistes et ainsi, de proposer des pistes visant à concilier ce qui paraissait impossible.

Cette perspective nécessite néanmoins beaucoup de prudence en raison du champ très large et à géométrie variable que représente le spectre autistique.

\section{Les Troubles du Spectre Autistique (TSA)}

4 Les troubles du spectre autistique sont encore très mal connus et souffrent d'un stéréotype oscillant entre une intelligence hors norme et une très forte agressivité, le plus souvent autodestructrice, et conduisant à l'idée d'un comportement imprévisible difficile à gérer.

Les TSA sont des troubles neurodéveloppementaux, c'est-à-dire qu'ils apparaissent durant la phase de développement de l'enfant. Le diagnostic d'autisme est posé lorsqu'on retrouve chez une même personne ce que l'on nomme la "dyade autistique » ${ }^{3}$, expression qui renvoie à deux symptômes majeurs: d'une part, les troubles de la communication sociale (difficultés ou impossibilités à verbaliser) et les troubles sociaux (problèmes de compréhension des codes de la société); il s'ajoute à cela des centres d'intérêt restreints et répétitifs (faire tourner une roue indéfiniment etc.) ${ }^{4}$.

Cependant, l'autisme ne se réduit pas à cette simple dyade. En effet, la complexité de l'autisme tient à la multitude de ses facettes. L'appellation de «troubles du spectre autistique » a d'ailleurs été choisie pour englober la très grande variété des troubles allant de pair avec l'autisme : d'un QI normal à une déficience intellectuelle en passant par une intelligence spécialisée (par exemple les "surdoués» en mathématiques, zoologie, histoire, etc.), les TSA, lorsqu'ils sont sévères, peuvent s'accompagner de difficultés motrices, de déficiences du langage, du comportement ou encore de l'attention etc. Ces troubles associés sont propres à chaque individu et ne suivent en aucun cas un schéma général.

6 Une forme de symptôme récurrent dans les TSA est les troubles sensoriels ${ }^{5}$. Ceux-ci peuvent varier en expression et en intensité au cas par cas. Leur prévalence selon les études, va de 30 à $100 \%$. Le pourcentage dépend essentiellement des méthodes choisies, mais globalement, les recherches convergent vers un pourcentage de $90 \%$ atteignant selon certaines études près de $100 \%$. Ces troubles affectent un ou plusieurs sens au travers de plusieurs organes sensoriels. Ils sont engendrés par une modification de la perception des stimuli sensoriels à cause d'un dysfonctionnement du traitement de l'information par le système nerveux central. 
7 Les troubles sensoriels associés à l'autisme peuvent se présenter de deux façons : une hypersensibilité aux stimuli produits dans l'environnement, ou à l'inverse, une hyposensibilité. L'une et l'autre se traduisent par une difficulté à gérer et traiter l'information sensorielle au quotidien, ce qui génère des situations de stress, d'anxiété, de colère et même de douleur physique. Ainsi, dans le cas d'une hypersensibilité auditive, le bruit d'un ballon qui éclate, une sonnerie d'alarme ou une annonce sonore peuvent engendrer une douleur auditive sévère ou au contraire, être recherchés comme une addiction dans le cas d'une personne hyposensible. Cette hyposensibilité peut se révéler dangereuse : la recherche d'un stimulus sensoriel trop important en termes de volume sonore entraînant parfois une surdité. Outre leur dangerosité relative, ces problèmes sensoriels génèrent des comportements atypiques, qui peuvent surprendre l'entourage social non averti, et particulièrement dans l'espace muséal. Ainsi les personnes avec un TSA hypersensible à certaines fréquences sonores en viennent à se boucher les oreilles dans l'espace public.

Fig. 1

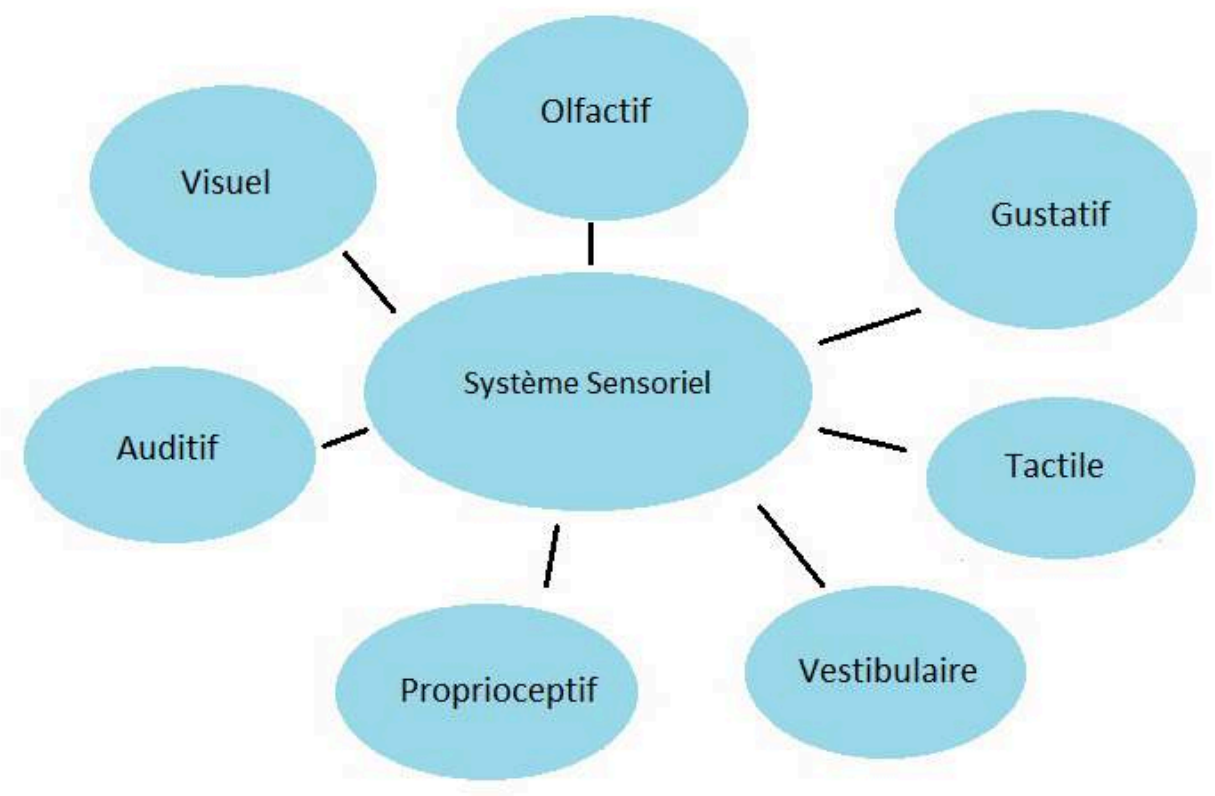

Schéma du système sensoriel, à partir de aspieconseil.com

(c) Cyrielle Leriche

8 L'autisme au sens large se caractérise par d'autres facteurs très importants dans la perspective d'une approche muséale :

Le cerveau des personnes autistes est essentiellement tourné vers une pensée en images. Comme l'écrit Temple Grandin ${ }^{7}$ dans son autobiographie : « Les autistes ont du mal à apprendre ce qui ne se traduit pas en images. Les mots les plus faciles à apprendre sont les noms parce qu'ils sont directement associés à une image ».

Ainsi, les personnes avec des TSA perçoivent le langage et les concepts associés sous forme d'images. À l'évocation du mot " porte » c'est une mini-vidéo de toutes les portes qu'ils ont pu voir dans leur vie qu'ils entrevoient et non le concept langagier de porte, avec son cortège de connotations et de métaphores associées. 

tour un obstacle à la compréhension de concepts généraux et à la manipulation de notions abstraites. Ainsi, devant une sculpture ou une peinture, les personnes avec des TSA s'attarderont sur des détails, parfois insignifiants pour les autres, ou judicieux et inattendus. En revanche, parler d' " esthétique » ou de "construction plastique » aura peu de signification.

10 Chez les personnes autistes, la vision périphérique est très développée, au point qu'elle supplante la vision focale. Si cela leur permet de mieux percevoir ce qui est présent autour d'elles, en revanche, elle a comme inconvénient majeur de limiter leur temps de concentration sur un objet donné.

11 La peur de l'imprévu constitue un trait important du profil psychologique des personnes autistes. Elle est à l'origine d'angoisses et de réactions de rejet. Pour toute sortie dans un lieu inconnu ou inhabituel, un travail préparatoire est primordial. Il consiste essentiellement à expliquer aux personnes en question ce qui va se passer, ce que l'on attend d'elles, mais aussi à leur présenter le plus clairement possible le lieu dans lequel elles vont se rendre.

12 La pluralité des principales facettes énoncées ci-dessus montre qu'il n'existe pas un autisme "type » mais une variété très importante de profils autistiques. Dans la perspective d'une approche de l'espace muséal, c'est une donnée importante à prendre en compte

\section{Autisme et nouvelles approches muséales}

13 Malgré la marginalisation des personnes avec autisme dans le cadre social comme muséal, un intérêt récent semble naître au sein des musées pour les personnes avec TSA. Les projets se multiplient aujourd'hui et l'on peut distinguer trois grandes thématiques: les dispositifs d'aide à la visite, le bien-être du visiteur et la visée thérapeutique. Si les deux dernières ne peuvent se concrétiser qu'à partir d'une visite réelle, la première est facilement mise en place à travers des sites internet propres à chacun des musées souhaitant accueillir des visiteurs avec TSA.

Dans cette optique, à travers le programme « MyMet ", le Metropolitan Museum of Art (MET) de New York $^{8}$ a mis à disposition des familles et des personnes concernées des indications permettant de faciliter leur venue au musée. Sur le site internet, des fichiers en format PDF peuvent être téléchargés. Ils offrent des conseils pour la visite aux parents ou aux porteurs directs des troubles. On y trouve également des fiches qui montrent des plans de galeries, des photos des salles d'exposition, ou encore, qui indiquent la qualité sensorielle des espaces ${ }^{9}$. Tous ces éléments représentent des aides aux visiteurs avec TSA, dans la mesure où ils préparent, planifient et rassurent sur le déroulé de la visite une fois sur place.

Le musée du Louvre à Paris s'est également investi dans ces dispositifs d'aide à la visite. En effet, outre l'accueil de publics provenant d'instituts médicaux éducatifs (IME), cet établissement a voulu repenser l'accès à la visite, tant pour les personnes autistes que pour les professionnels enseignants. Il a sollicité l'aide d'une éducatrice spécialisée, Catherine Treese-Daquin ${ }^{10}$ laquelle a ainsi proposé des fiches actuellement consultables sur le site du musée du Louvre permettant d'organiser une visite ${ }^{11}$. Ces fiches se focalisent sur quelques lieux précis : la Cour Khorsabad, le Louvre médiéval, ainsi que 
sur les chefs-d'œuvre du musée. Elles rappellent les objectifs de la visite et sélectionnent des œuvres avec leurs explications. On retrouve également dans ces fiches abondamment illustrées une liste de matériel pouvant être nécessaire à la visite (carnet et crayon pour dessiner) mais également des supports visuels à imprimer qui facilitent grandement la compréhension pour un public autiste plus visuel qu'un public neurotypique ${ }^{12}$.

Ces aides préliminaires à la visite représentent un outil primordial, car elles contribuent à atténuer la peur de l'inconnu et celle de l'imprévu dans la pensée des personnes concernées. En suggérant en amont une visite attractive, ludique et viable avec des dispositifs sensoriels, elles ont pour effet de diminuer l'angoisse, source constante d'échec.

Lors de la visite elle-même, il s'agit d'entretenir et de consolider le regard positif porté par ces aides préliminaires. Certains musées se sont donnés pour objectif premier de traiter le sentiment de bien-être. Si cet objectif concerne bien entendu tout public, il est servi par des programmes particulièrement plus adaptés pour les personnes avec autismes

18 Il en est ainsi du programme appelé "Exploring our way " proposé au Children's Museum de New Hampshire ${ }^{13}$ tous les premiers dimanches du mois. Les enfants autistes, obligatoirement accompagnés d'un adulte, peuvent arriver jusqu'à deux heures avant l'ouverture officielle au public, prévue à midi, et sont encouragés à rester après l'heure de fermeture s'ils le souhaitent. Deux dispositifs caractérisent le programme, tous deux fondés sur le langage visuel: le MAPPS (Museum Advanced Planning Picture) et le PECS (Picture Exchange Communication System). Le premier est un outil pédagogique qui consiste à formuler des phrases où chaque mot est illustré par un dessin. Le second dispositif, le PECS est un outil de communication qui existe depuis près de 20 ans aux États-Unis, fondé sur un système d'échange d'images. Cette méthode fonctionne particulièrement bien pour les enfants et adultes non verbaux. Les dessins ont valeur de signe et de logo qui permettent par exemple de transmettre une consigne, une tâche à faire ou d'exprimer des sentiments (peur, colère, tristesse), un système que l'on peut rapprocher par certains côtés des émoticônes. Ici, le programme mise sur le bien-être des participants pour les familiariser avec le lieu et les inciter à rester, à revenir.

Certains musées ambitionnent même une visée thérapeutique au sein de leurs programmes.

Le musée des Beaux-Arts de Montréal fait ainsi figure de pionnier grâce à son programme "L'art dans tous ces états ${ }^{14}$ où l'art devient un espace potentiel d'épanouissement en transformant un moment a priori stressant en une visite vécue positivement et ressentie comme telle. Ce programme permet aux participants de tirer profit des collections pour approfondir leurs connaissances des émotions et des comportements sociaux en s'appuyant par exemple sur les scènes représentées dans les œuvres. La visée thérapeutique réside dans l'expression orale et la prise de parole des participants qui sont incités à émettre des jugements critiques. Cette libération de la parole est parachevée par un atelier d'art-thérapie avec une réalisation personnelle.

21 Le New York Transit Museum s'investit également dans cette dimension thérapeutique, à travers le programme "Subway Sleuths $»^{15}$. Ce programme parascolaire exploite l'intérêt commun des autistes pour les trains afin d'encourager l'interaction et de développer les aptitudes sociales. Les participants répartis en binômes explorent les 
reproductions de stations de métros, résolvent des mystères, deviennent des experts du transport en commun tout en partageant leurs enthousiasmes. Chaque classe est animée par un professeur d'éducation spécialisée, un orthophoniste et un médiateur du musée. La démarche est très intéressante, car elle se sert d'un centre d'intérêt très présent dans l'autisme (les transports ou plus précisément la mécanique) pour motiver et faire travailler les enfants en groupe.

Ces deux expériences offrent avant tout une prise en charge collective des visiteurs atteints de troubles autistiques et nécessitent la présence d'encadrants spécialisés. Néanmoins, les participants sont en quelque sorte isolés de l'univers réel du musée, reconnaissant l'incapacité pour une personne autiste à effectuer une visite de façon autonome. Une fatalité à laquelle le Victoria \& Albert Museum a tenté de répondre. À la suite d'un long travail de collaboration avec des familles dont un membre était atteint de TSA. Le musée a conçu des sacs à dos "sensoriels" pouvant être empruntés gratuitement par les visiteurs concernés. Chaque sac contient des cartes, des accessoires à toucher, des suggestions d'activités et des protèges oreilles permettant de diminuer le bruit ambiant. Cet équipement présente l'avantage de favoriser une certaine autonomie et d'intégrer les personnes autistes dans le flux normal des visiteurs. Surtout, il prend en compte un obstacle majeur, celui de l'inconfort sensoriel éprouvé par les personnes autistes lorsqu'elles se trouvent dans un espace public à forte concentration humaine. C'est dans cette perspective que nous avons mené une expérience au sein du Musée du Louvre afin de mesurer l'impact de l'environnement sensoriel sur la réception et la compréhension des discours de médiation.

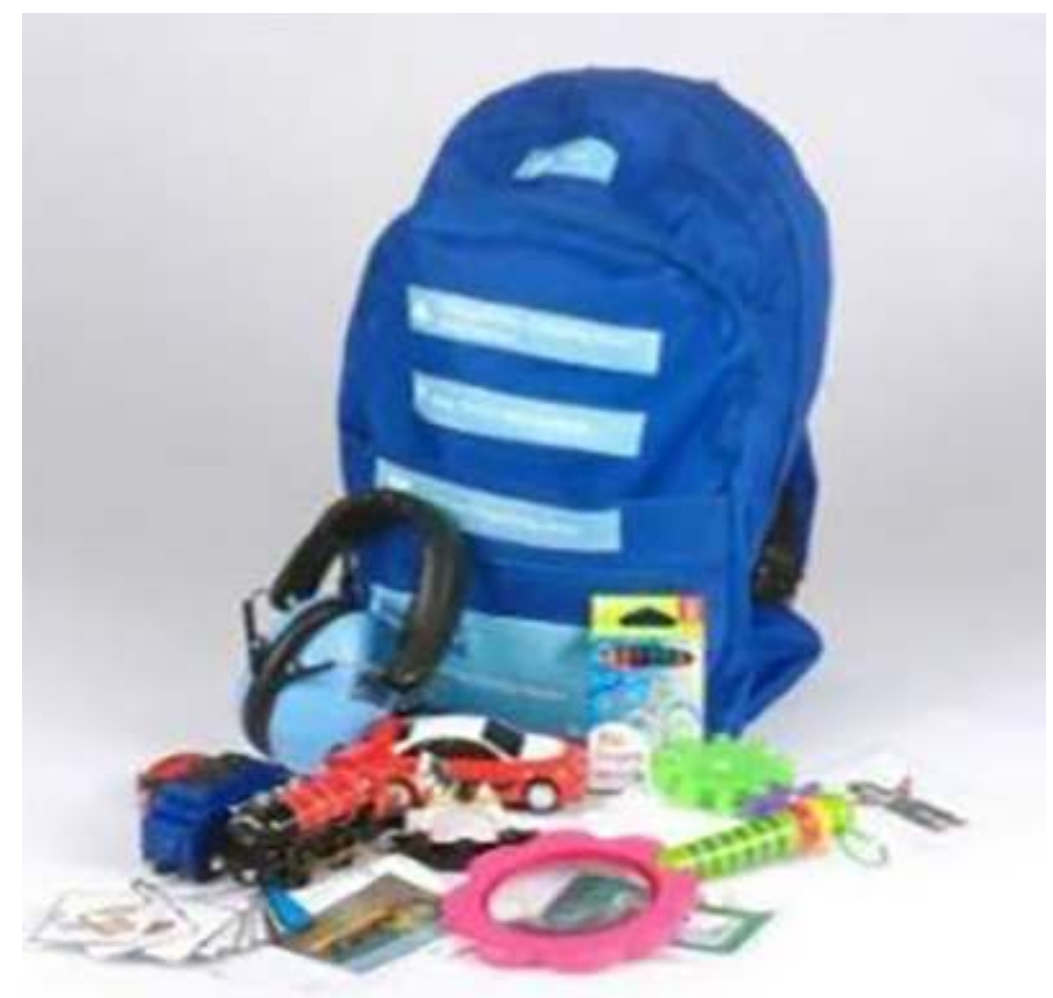

Sac à dos sensoriel du Victoria \& Albert Museum (c) Victoria and Albert Museum, Londres 


\section{L'impact de l'environnement sensoriel dans le musée : étude préliminaire}

Cette recherche sur l'environnement sensoriel muséal vise un public atypique qui, bien que classé " autiste ", présente des profils à fort degré de variabilité. C'est pourquoi le choix du lieu, la constitution du groupe participant ainsi que la sélection des œuvres ont fait l'objet d'une réflexion nourrie de critères, certes arbitraires, mais opérationnels.

Si tous les participants admis pour cette étude devaient obligatoirement présenter un trouble du spectre autistique diagnostiqué par un médecin, n'ont été retenus que des individus présentant un TSA sans retard mental ni déficience de langage ${ }^{16}$. En effet, l'étude portant exclusivement sur l'impact de l'environnement sensoriel du musée sur le public TSA, il fallait recourir à des personnes en capacité d'exprimer verbalement ce qu'elles ressentaient. Ainsi, l'absence de retard mental et de déficience langagière permettait aux sujets de comprendre, de participer et surtout de répondre aux questionnaires. Un deuxième critère, l'âge des participants, fut également établi, sur une fourchette assez large. Afin de créer des ensembles homogènes, ils ont été répartis dans deux groupes : l'un comprenant 10 adolescents ${ }^{17}$ âgés de 10 et 19 ans $^{18}$; l'autre composé de 10 adultes âgés de 20 à 50 ans. Cette répartition présentait l'intérêt de déterminer si l'âge était un paramètre pertinent pouvant affecter la réception des stimuli sensoriels en musée, pour des personnes présentant des TSA. La limite de 50 ans a été arbitrairement choisie afin que ce deuxième groupe ait une homogénéité relative au niveau des capacités physio-perceptives.

Le choix du Louvre s'est imposé rapidement en raison de la multiplicité des espaces, chacun doté de paramètres spécifiques (fréquentation du public, éclairage, nature des collections, odeurs, température, résonance sonore...) De plus, le fait que le musée du Louvre soit un musée très célèbre et parfois même idolâtré a été un avantage dans le recrutement des participants, ravis de pouvoir passer un temps dans ce lieu prestigieux. Le département des antiquités grecques est apparu très propice pour mener les séances expérimentales. Parmi les cinq salles qui le composent ${ }^{19}$, celle des antiquités grecques préclassiques fut retenue en raison de sa localisation géographique au sein du musée et de son taux de fréquentation. En effet, cette salle est située juste à côté de l'entrée Denon, ce qui permet d'accéder rapidement à la visite sans avoir besoin de traverser tout le musée (cela pouvant être source de fatigue). Elle est également proche de la sortie en cas de besoin. De plus, la salle d'épigraphie, mitoyenne à la salle des antiquités grecques préclassiques, souvent déserte, offre au visiteur un lieu calme pour commencer la visite, répondre aux questionnaires mais également pour se reposer en cas de crise ou de trop pleins sensoriel.

Les œuvres présentées ont également fait l'objet d'une sélection précise. L'art grec est intéressant car il peut être abordé de différentes façons selon les capacités cognitives et intellectuelles du sujet: histoire de l'art, histoire de la civilisation, mythologie. Afin d'éviter une trop grande fatigue des participants et de rentrer dans les 45 minutes de visite prévues, il était impératif de présenter, au maximum, six œuvres durant la visite. Les œuvres ont été choisies de manière à présenter différents niveaux de difficultés de lecture et d'interprétation: 

sculptures monumentales de marbre représentant le corps humain. La première sculpture est une statue féminine de la période archaïque (VII-VIe siècle av. J.-C.), la seconde est un torse nu d'homme du début de la période classique (V-IVe siècle av. J.C.). Les deux œuvres baignent dans deux environnements sensoriels très différents : la Korê se situe dans la salle de la Grèce préclassique avec un éclairage naturel, une température similaire aux autres œuvres et un flux de visiteurs moindre, tandis que le Torse de Milet, se situe dans un couloir de transition permettant de prendre les escaliers montant à la salle des antiquités grecques classiques. L'environnement sensoriel de cette salle change totalement; il y fait sombre avec un éclairage faible, artificiel et jaunâtre, un courant d'air, un flux de visiteurs bien plus important et un brouhaha émanant du haut de l'escalier.

31 L'amphore bilingue des peintres d'Andokidès et de Lysippidès présente la scène $d u$ dernier des douze travaux d'Héraclès, la capture de Cerbère (chien des enfers) sur sa face $\mathrm{A}$ et une scène dionysiaque sur sa face $\mathrm{B}$. Cette œuvre est intéressante car les personnes avec TSA ont du mal à comprendre et à interpréter des scènes, à les intégrer mentalement, se confrontant plus aux textures ou aux touches du peintre, c'est-à-dire, à des éléments concrets, réalistes et visuels.

32 Ajoutons également que ce choix d'œuvres permet d'aborder l'art grec sous différents angles: la mythologie, la civilisation, les techniques de créations et l'histoire de l'art. Toutes ces approches étaient essentielles pour correspondre aux différentes attentes des vingt participants et pour voir l'art grec sous des aspects qui leur étaient probablement inconnus.

Les visites ont été présentées aux participants et à leurs accompagnants une semaine à l'avance au travers d'un document récapitulant les informations pratiques et comprenant surtout, outre des indications écrites et visuelles du lieu de la visite, de la station de métro et du lieu de rendez-vous, une photo du médiateur. Le déroulé du programme avec quelques images d'œuvres grecques différentes de celles prévues lors de la séance complétait le document. L'objectif était de ne pas laisser de place à l'imprévu.

Ce dispositif préparatoire comprenait également un questionnaire sensoriel tiré du Profil sensoriel de Winnie Dunn ${ }^{20}$. Ce questionnaire propose aux participants autistes d'évaluer eux-mêmes leur niveau de gêne sensorielle dans leur vécu quotidien, selon trois degrés : une "performance typique» (niveau de gêne sensorielle "normale»), 
une «différence probable " (niveau de gêne sensorielle marqué) ou une "différence avérée » (niveau de gêne sensorielle très important) du vécu sensoriel. Cette donnée est apparue nécessaire pour mesurer le degré de gêne ressentie engendrée par l'environnement quotidien. Cette mesure, bien que relevant pour une part du subjectif, permettait par la suite d'avoir une référence pour comparer la gêne induite par la sensorialité au quotidien à celle induite à l'instant $\mathrm{T}$ de la visite.

\section{Déroulement des séances} l'histoire grecque, sa chronologie et ses inventions, avant de passer à la première œuvre. Les six œuvres étaient ensuite présentées à raison de cinq à six minutes chacune. Quelques points majeurs étaient soulevés, toujours appuyés d'une carte géographique et d'une frise chronologique afin de replacer les données spatiotemporelles, parfois difficiles d'accès. Le discours a été conçu comme un échange, une conversation, une visite interactive et non uniquement comme une dispense de savoir. Cette interaction est absolument nécessaire car l'implication active d'un visiteur, notamment autiste, permet d'avoir son attention avec plus de facilité. De même, le commentaire des œuvres était constamment accompagné de supports visuels: des photos de détails et d'angles de vue différents, des reproductions d'œuvres comparables. Ces supports visuels se sont avérés indispensables au bon déroulement des séances. Il fallait représenter tout ce qu'ils ne pouvaient pas voir et faire en sorte que chaque propos puisse susciter une adéquation avec une pensée en image, et par là même une compréhension aboutie. Les participants étaient invités systématiquement à poser des questions à la fin de chaque présentation. Cela est tout à fait banal dans le cas d'un public classique, mais lors de ces séances, et bien qu'il s'agisse de séances individuelles, la plupart des sujets n'osaient pas poser de question tant qu'on ne les y invitait pas. D'une façon générale, la vigilance était renforcée. Il fallait être très attentif 
à leurs comportements durant la visite : voir ce qui les troublait, s'arrêter de parler quand une question était latente et les encourager quand ils cherchaient une réponse ou au contraire quand ils en donnaient une mauvaise, ce qui pouvait être très frustrant pour eux. Pour exemple, lors d'une visite avec un adolescent qui avait donné plusieurs mauvaises réponses, la frustration, l'agacement et l'énervement commençaient à se traduire dans son comportement. Il a donc fallu l'encourager en rebondissant sur ses réponses et en le stimulant pour la suite de la visite.

À la fin de la visite, deux nouveaux questionnaires ont été présentés aux participants, questionnaires créés spécialement pour cette recherche. Le premier consistait à évaluer la compréhension de la visite. Un temps de 15 minutes était prévu afin que les participants puissent y répondre. Pour les participants du groupe adulte, il s'agissait d'un questionnaire simple. Pour les participants les plus jeunes, le questionnaire était sous forme de livret-jeu. À noter cependant que les deux supports sont très visuels, avec une photo associée à chaque question posée. Le questionnaire est composé uniquement de questions fermées (avec choix de propositions). Le fait que ces questions soient alimentées de deux propositions à chaque fois permet de pallier la peur de l'échec, tant pour l'adolescent que pour l'adulte, qui pouvait les pousser à ne pas répondre du tout. À la fin de ce premier questionnaire se trouvait une question ouverte, sans obligation de réponse, pour que les participants puissent s'exprimer librement. Les questions posées portaient sur la typologie, l'utilisation, l'iconographie ou encore l'histoire des œuvres.

Le deuxième questionnaire analyse le vécu sensoriel en musée afin de savoir ce qui a gêné, ou non, les participants. Ce questionnaire se présente sous la forme d'une échelle colorée (avec scratch adhésif) allant de " pas du tout gêné » à " extrêmement gêné » sur laquelle les participants doivent, eux-mêmes, scratcher les éléments qui ont pu les gêner, ou pas, en musée tels que la lumière, le bruit ou encore le positionnement des œuvres. Cela permet également d'observer la différence de gêne sensorielle entre celle du quotidien et celle engendrée par le musée au regard du Profil Sensoriel de Winnie Dunn.

La fin de la visite était propice à une ouverture sur le musée. Il leur était toujours demandé s'ils désiraient rentrer chez eux ou bien poursuivre une visite libre dans le musée, avec ou sans le médiateur. Ce temps de découverte a très bien marché puisque $50 \%$ des participants ont souhaité rester au musée, systématiquement avec le médiateur. 
Fig. 3

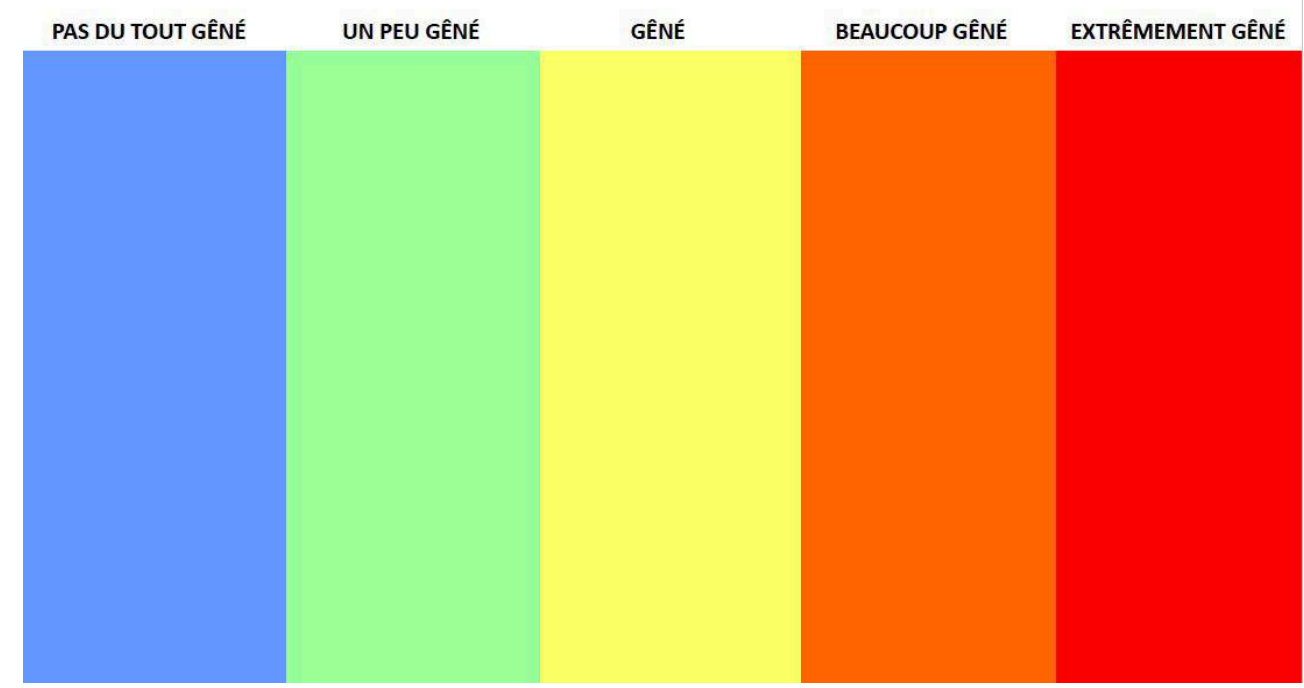

Échelle sensorielle colorée permettant de classer les items ci-dessous en fonction du niveau de gêne en musée.

(c) Cyrielle Leriche

Fig. 4

\begin{tabular}{|c|c|c|c|}
\hline VISUEL & AUDITIF & TACTILE & OLFACTIF \\
\hline LUMIERE DES CEUVRES & $\begin{array}{l}\text { BRUIT DE FOND } \\
\text { (VISITEURS...) }\end{array}$ & $\begin{array}{l}\text { CONTACT AVEC LES } \\
\text { VISITEURS }\end{array}$ & ODEURS DU MUSEE \\
\hline LUMIERE DE LA SALLE & & & ODEURS DES VISITEURS \\
\hline $\begin{array}{l}\text { DISPOSITION DES GEUVRES } \\
\text { DANS LA SALLE }\end{array}$ & $\begin{array}{l}\text { BRUIT INATTENDU } \\
\text { (ANNONCES...) }\end{array}$ & TEMPERATURE DE LA SALLE & \\
\hline $\begin{array}{l}\text { CEUVRES DE GRANDES } \\
\text { TAILLES }\end{array}$ & & $\begin{array}{l}\text { ORGANISATION DE LA } \\
\text { SALLE }\end{array}$ & \\
\hline \multicolumn{4}{|l|}{$\begin{array}{l}\text { CEUVRES DE PETITES } \\
\text { TAILLES }\end{array}$} \\
\hline \multicolumn{4}{|l|}{$\begin{array}{c}\text { COULEURS ET FORMES DES } \\
\text { CFUVRES }\end{array}$} \\
\hline $\begin{array}{l}\text { ๔EUVRES REGROUPEES } \\
\text { } \text { EUVRES ISOLEES }\end{array}$ & & & \\
\hline VITRINES & & & \\
\hline
\end{tabular}

Items sensoriels classés par catégories

(c) Cyrielle Leriche

\section{Analyse et bilan}

41 Les éléments d'observation relevés pour chacune des 20 séances et l'analyse des questionnaires rendus ont fait ressortir plusieurs points, en particulier, que la sensorialité n'avait pas forcément d'impact sur la compréhension du discours chez les adultes comme chez les adolescents. En revanche, les troubles cliniques associés se sont révélés très importants, influant sur l'expression langagière et la concentration. Pour exemple, un sujet pensant voir des dieux grecs lors de la visite au musée fut très 
perturbé et très peu attentif demandant sans cesse où étaient les représentations de divinités. Ce même sujet eut d'ailleurs le moins bon niveau de compréhension du groupe.

Un second élément qui ressort parfaitement est le rôle joué par le confort de visite, lequel mettait chaque participant dans un état d'esprit positif avant et durant la séance, abaissant ainsi leur seuil de sensibilité aux conditions environnementales (bruit, lumières...)

Les réponses au questionnaire de vécu sensoriel montrent que l'âge des participants n'a eu que très peu d'incidence. Les différences entre les deux groupes, «adolescents » et "adultes", ne sont pas significatives bien que les adolescents aient rencontré légèrement plus de difficultés à gérer la sensorialité en musée. Cela prouve une fois de plus qu'il n'est pas possible de classer les personnes TSA selon des clichés tels que l'âge mais que la différence se lit bien plus au niveau du profil psychologique de l'individu. Toutefois, le fait que les sujets ne présentaient pas énormément de difficultés sensorielles en musée peut s'expliquer par le fait que les participants n'avaient pas de retard mental et surtout qu'ils s'exprimaient verbalement. Ces deux paramètres les auraient rendus plus aptes à contrôler leur environnement sensoriel ou à mettre en place des stratégies d'adaptation.

Un autre facteur ayant pu minimiser la gêne sensorielle semble avoir été déterminant : la durée restreinte dans le temps de la séance et le nombre réduit des œuvres commentées donnaient lieu à un rythme rapide ce qui permettait de retenir l'attention et de favoriser une durée de concentration plus longue. Cette explication va tout à fait dans le sens d'une étude menée en 2017 par Fern Silverman et Andrea Carr Tyska concluant que les programmes spécialisés avec les modifications appropriées améliorent la qualité et la durée de la visite en musée pour des personnes TSA avec des troubles sensoriels ${ }^{21}$.

Enfin, les analyses de ces questionnaires ont également mis en avant que les éléments sensoriels les plus problématiques en musée sont liés aux sonorités inattendues, à l'intensité des bruits de fond et aux contacts avec les visiteurs. Or, ces éléments constituent l'essence même des musées à forte fréquentation : un microcosme social où un flux de visiteurs bruyants et nombreux se disperse dans les salles du musée. Ces deux éléments, le bruit et l'affluence de personnes, sont également ceux qui ressortent le plus du profil sensoriel de Dunn.

Il n'est donc évidemment pas possible de créer une visite type en musée pour les personnes avec autisme mais il est indispensable de privilégier l'adaptation selon chaque personne pour chaque visite.

\section{Des pistes à explorer}

47 Au regard des résultats de cette recherche, il serait évidemment tentant d'orienter les visiteurs à profil autistique vers des musées à faible taux de fréquentation. Cette proposition serait un aveu de renoncement et irait à l'encontre des préconisations actuelles qui incitent plutôt à l'intégration sociale des personnes autistes, et non à leur isolement ${ }^{22}$.

D'autres pistes pourraient être envisagées. Tout d'abord, il semble primordial que les musées indiquent les horaires et jours d'ouverture ayant une affluence moindre sur 
leurs sites internet. Toutefois, pour que ces visites soient possibles, il serait bienvenu que les musées proposent un système d'entrée dédiée sans queue ni turbulence; une attente trop longue, statique, dans une foule noyée par les impulsions sonores étant une invitation à rebrousser chemin.

Afin de susciter les visites spontanées des familles concernées, la mise en ligne de supports d'aides à la visite comprenant des fiches adaptées semble avoir fait leurs preuves, comme l'ont démontré les expériences menées au Metropolitan Museum et le Museum of Modern Art de New York ou au musée du Louvre ${ }^{23}$ de Paris. La préparation en amont étant bien souvent déterminante pour assurer le bon déroulement de la visite et inciter ces visiteurs atypiques à revenir.

On peut également imaginer, un peu comme au Children's Museum de New Hampshire, que certains musées instaurent selon leurs possibilités des temps calmes un jour ou deux par semaine avec un accès réservé et des espaces moins chargés (diminution des lumières et $\mathrm{du}$ fond sonore etc.). Cependant, cette proposition ne doit être prise comme unique possibilité d'accueil d'un public avec autisme au sein des institutions muséales. En effet, elle a pour inconvénient d'être excessive, si ce n'est contre-productive. Elle aurait pour conséquence d'isoler encore plus les personnes autistes du tissu social. Or, il s'agit avant tout de considérer le musée comme un facteur potentiel d'intégration dans le monde ordinaire. C'est pourquoi tout ce qui peut favoriser cette intégration doit être mis en avant. Il en est ainsi des sacs sensoriels du Victoria \& Albert Museum qui semblent être des outils ingénieux pour le bon vécu de la visite. En plus d'offrir une activité ludique, ils rendent la visite supportable et vivable grâce notamment aux casques à diminution de bruit (tels que ceux préconisés par la plupart des psychomotriciens pour les enfants autistes lors de leurs séances). Ces sacs pourraient être enrichis d'autres dispositifs comme, par exemple, des lunettes de soleil pour les salles trop lumineuses.

51 Les vingt séances ont confirmé l'importance de la visite individualisée, préférable à la visite de groupe. Dans ce cadre, une formation spécifique des médiateurs sur les troubles du spectre autistique est indispensable, ne serait-ce que pour ne pas être dépassé en situation de crise, ou plus simplement pour éviter certains comportements inadéquats, comme regarder avec insistance droit dans les yeux, toucher la personne, et accepter un temps de réflexion plus ou moins long entre la question posée et la réponse. Cette formation préalable ne remplacera pas l'expérience d'un psychothérapeute et elle ne doit surtout pas faire l'économie de la présence d'un(e) accompagnant(e).

\section{Conclusion}

52 Au terme de cette étude, il apparaît clairement que la relation antinomique pressentie entre le monde des musées et les personnes autistes ne relève pas d'une fatalité. Les institutions muséales sont des lieux privilégiés d'intégration sociale pour les personnes avec un trouble du spectre autistique: lieu d'Histoire, de savoir, de culture mais également de partage, d'expérience sociale et sensorielle : les musées constituent un fabuleux lieu d'émerveillement et d'apprentissage.

Cette perspective n'implique pas une transformation radicale du musée, mais plutôt une démarche qui vise à inventer, adapter et à développer des dispositifs accentuant le confort sensoriel de ces visiteurs atypiques. Cela représente un véritable défi au regard 
de la diversité des facettes que revêt le spectre des troubles autistiques. L'augmentation alarmante du nombre de cas diagnostiqués "autistes" au cours des dernières décennies $^{24}$ a suscité une prise de conscience justifiée de la part des organismes concernés. Nul doute que l'institution muséale pourra apporter une contribution originale et pertinente.

\section{NOTES}

1. Notamment avec la création de l'Observatoire Permanent des Publics en 1989.

2. Voir « Autisme et nouvelles approches muséales » (deuxième partie).

3. American Psychiatric Association, DSM-5, Manuel diagnostique et statistique des troubles mentaux ("Diagnostic and Statistical Manual of Mental Disorders"), Édition Elsevier Masson, 2013.

4. Ibidem.

5. Danièle Caucal et Régis Brunod, Les aspects sensoriels et moteurs de l'autisme, AFD édition, 2010.

6. Claire Degenne-Richard, Marion Wolff, Dominique Fiard, Jean-Louis Adrien, «Les spécificités sensorielles des personnes avec autisme de l'enfance à l'âge l'adulte ", ANAE - Approche Neuropsychologique des Apprentissages de l'Enfant, $\mathrm{N}^{\circ}$ 128, 2014.

7. Temple Grandin, Penser en image et autres témoignages sur l'autisme, Odile Jacob, 1995.

$8 . \quad$ https://www.metmuseum.org/events/programs/access/visitors-withdevelopmental-and-learningdisabilities/for-visitors-with-autism-spectrum-disorders

9. La fiche indique par exemple le degré de luminosité des salles et le degré d'affluence du public.

10. Catherine Treese-Daquin est une éducatrice spécialisée dans l'autisme et personne relais au musée du Louvre.

11. https://www.louvre.fr/pistes-de-visite/aux-origines-du-louvre-le-louvre-medieval

12. Neurotypique : personne n'ayant pas de trouble du spectre autistique.

13. https://www.childrens-museum.org/things-to-do/programs/autism

14. http://autismesanslimites.org/partenariat-musee-des-beaux-arts-de-montrealautisme-sans-limites/

15. http://www.nytransitmuseum.org/learn/subwaysleuths/

16. Depuis le DSM 5, les appellations d'«Asperger» ou d'« Autisme à haut niveau de fonctionnement » ne sont plus en vigueur.

17. Selon les critères de l'OMS: "L'adolescence est la période de croissance et de développement humain qui se situe entre l'enfance et l'âge adulte, entre les âges de 10 et 19 ans. »- http://www.who.int/maternal_child_adolescent/topics/adolescence/dev/fr/

18. Les enfants n'ont pas été admis dans cette étude car leurs niveaux de compréhension auraient été trop bas, biaisant ainsi les résultats de l'étude. 
19. La salle des antiquités grecques classiques, la salle des antiquités grecques hellénistiques, la salle des bronzes et la galerie Campana.

20. Dunn Winnie, Profil Sensoriel, CPA, Éditions du centre de psychologie appliquée, 2010 (version abrégée).

21. SILVERMAN Fern et CARR TYSKA Andrea, Supporting Participation for Children With Sensory Processing Needs and Their Families : Community-Based Action Research, mai 2017

22. Rita Jordan et Stuart Powell, Les enfants autistes: les comprendre, les intégrer à l'école, Edition Masson, 1997.

23. Catherine Treese-Daquin, Autisme et Culture - Balade au musée du Louvre, AFD édition, février 2016.

24. $\quad$ https://www.has-sante.fr/upload/docs/application/pdf/2010-03/ autisme__etat_des_connaissances_synthese.pdf

\section{RÉSUMÉS}

La politique muséale tend actuellement à prendre en charge les publics dits "spécifiques". Toutefois, une forme de handicap reste très marginalisée du monde des musées : les troubles du spectre autistique. L'autisme est encore très mal connu du grand public dont la connaissance prend, trop souvent, racines dans des idées reçues. La venue des personnes avec autisme dans les institutions culturelles est fortement marquée par cette exclusion. L'absence de ce public des salles muséales peut s'expliquer de plusieurs façons et, notamment, par un élément caractéristique du musée: son environnement sensoriel. Cette étude a pour objectif de démontrer l'impact de l'environnement sensoriel du musée sur les visiteurs avec autisme au travers d'une expérimentation de terrain afin de proposer une adaptation du musée favorable à la venue de ce public.

Museum policy currently welcomes disabled visitors. However, one type of disability remains very marginalised in the museum world: autistic spectrum disorder. Autism is still somewhat of a mystery to the general public whose knowledge of it is too often rooted in commonplaces. The visits to cultural institutions by people with autism is strongly marked by this exclusion. The absence of autistic visitors from museums may be explained in many ways, particularly by an element characteristic of the museum: its sensorial environment. This study aims to demonstrate the impact of the sensorial environment of the museum on visitors with autism through a field study in order to propose an adaptation of the museum favourable to the visits of those with autism.

\section{INDEX}

Mots-clés : accessibilité, handicap, autisme, sensorialité, médiation, expérimentation

Keywords : accessibility, handicap, autism, sensory perception, mediation, field study 


\section{AUTEUR}

\section{CYRIELLE LERICHE}

Après un premier cycle à l'École du Louvre spécialisée en art et archéologie du monde grec, option épigraphie grecque, Cyrielle Leriche s'est intéressée aux publics spécifiques durant son deuxième cycle. Travaillant lors de son année de Master 1 sur l'évaluation d'un programme destiné aux personnes atteintes de la maladie d'Alzheimer du musée du Louvre, ce deuxième mémoire a été l'occasion d'ouvrir une porte sur la prise en charge des personnes présentant un trouble du spectre autistique dans les musées.

After a first degree at the École du Louvre specialising in the art and archaeology of the Greek world with an option in Greek epigraphy, Cyrielle Leriche became interested in disabled museum visitors during her postgraduate work. Her Master 1 work on the evaluation of a programme for people suffering from Alzheimer's at the Musée du Louvre opened a door on to the ways museums deal with visitors with autistic spectrum disorder. 